\title{
An optimal state estimation model of sensory integration in human postural balance
}

\author{
Arthur D Kuo \\ Department of Mechanical Engineering, University of Michigan, 2350 Hayward St., Ann Arbor, \\ MI 48109-2125, USA \\ E-mail: artkuo@umich.edu
}

Received 4 March 2005

Accepted for publication 13 July 2005

Published 31 August 2005

Online at stacks.iop.org/JNE/2/S235

\begin{abstract}
We propose a model for human postural balance, combining state feedback control with optimal state estimation. State estimation uses an internal model of body and sensor dynamics to process sensor information and determine body orientation. Three sensory modalities are modeled: joint proprioception, vestibular organs in the inner ear, and vision. These are mated with a two degree-of-freedom model of body dynamics in the sagittal plane. Linear quadratic optimal control is used to design state feedback and estimation gains. Nine free parameters define the control objective and the signal-to-noise ratios of the sensors. The model predicts statistical properties of human sway in terms of covariance of ankle and hip motion. These predictions are compared with normal human responses to alterations in sensory conditions. With a single parameter set, the model successfully reproduces the general nature of postural motion as a function of sensory environment. Parameter variations reveal that the model is highly robust under normal sensory conditions, but not when two or more sensors are inaccurate. This behavior is similar to that of normal human subjects. We propose that age-related sensory changes may be modeled with decreased signal-to-noise ratios, and compare the model's behavior with degraded sensors against experimental measurements from older adults. We also examine removal of the model's vestibular sense, which leads to instability similar to that observed in bilateral vestibular loss subjects. The model may be useful for predicting which sensors are most critical for balance, and how much they can deteriorate before posture becomes unstable.
\end{abstract}

(Some figures in this article are in colour only in the electronic version)

\section{Introduction}

The human central nervous system (CNS) regulates postural balance of a complex, multi-segment body with many actuators and a wide array of sensors. Each of these sensors provides localized and imperfect information regarding the motion of one or more body segments, subject to the dynamics of the sensors themselves. These data are then conveyed to the CNS by an array of afferents, each of which provides only a fraction of the total sensory information. The CNS continuously generates motor commands to compensate for the unstable dynamics of the body. From a control systems standpoint, there are two challenging issues in posture. The first is the use of feedback to control an unstable system. The second is the processing of noisy sensory data to provide the information necessary to perform this control.

Upright standing places the body in an unstable configuration that requires active feedback stabilization (see figure 1(a)). The minimal set of information necessary to stabilize the body appears to be the full dynamical state, that is, data equivalent to the positions and velocities of all body segments. Inertial coupling between body segments 
(a) General Feedback Control Model

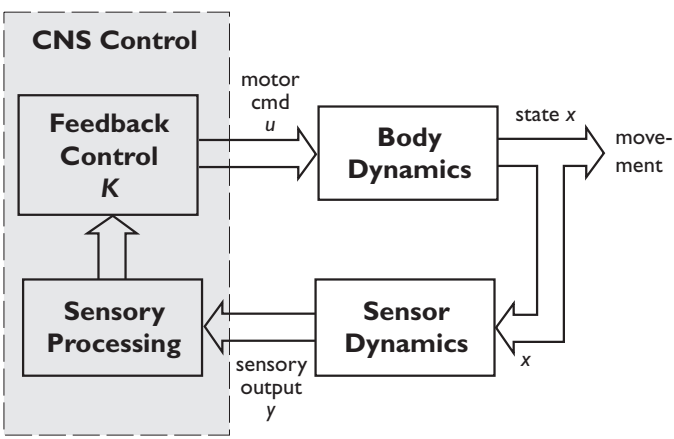

(b) Sensor Model

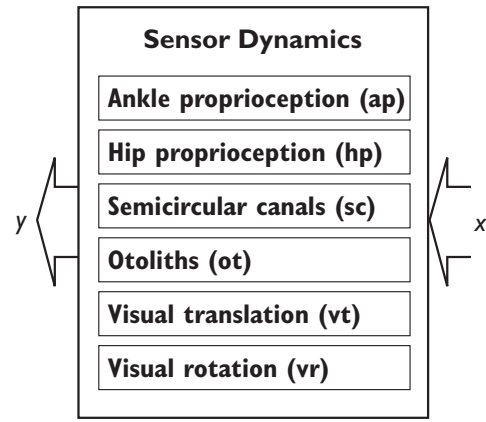

(c) Direct Feedback Model

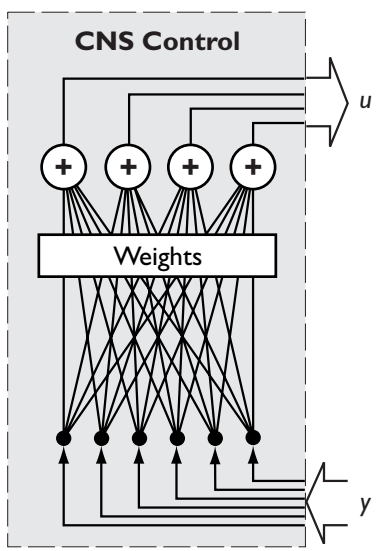

(d) State Estimator Model

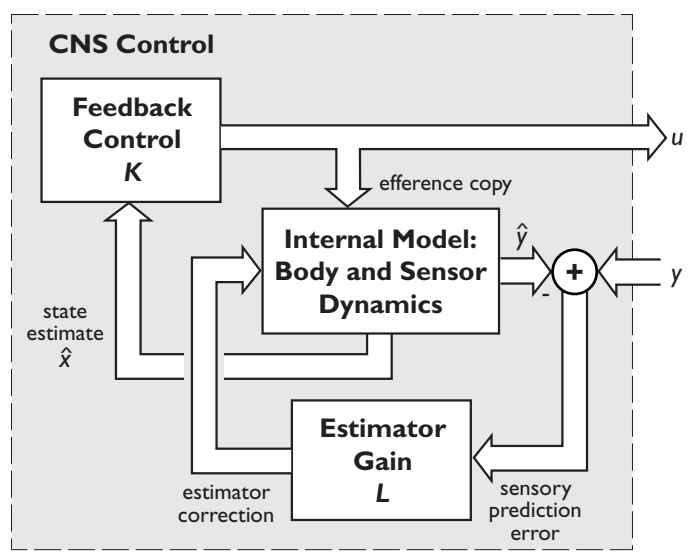

Figure 1. Schematics of central nervous system (CNS) control. (a) General feedback model produces motor commands $u$ that drive the body dynamics, producing motion described by body state $x$. Sensory dynamics translate the state into sensory outputs $y$, feeding back to the CNS. Feedback control $K$ is modeled as state feedback, preceded by sensory processing if necessary. (b) Sensors for posture are of three types: proprioceptors, vestibular sensors and vision. Major proprioceptors include ankle and hip muscle spindles; vestibular sensors include semicircular canals and otoliths; vision includes sensation of translation and rotation of the head. Each of these sensors has dynamics that temporally filter the state $x$. (c) Direct feedback is a simple model of feedback control where sensory outputs $y$ are fed directly into a matrix of gains to produce a motor command $u$ that is essentially a weighted sum of the components of $y$. (d) State estimation is a different method of sensory processing that uses an internal model of body and sensor dynamics and efference copy to produce the state estimate $\hat{x}$ that enters the feedback control gain matrix. The internal model also predicts the sensory output $\hat{y}$, and the error of this prediction is used to dynamically correct $\hat{x}$. State estimation can temporally process information from multiple sensors, each with distinct dynamics, so that disparate and noisy data can be integrated to yield an optimal estimate.

appears to make purely local feedback — controlling each joint with information only from that same joint—an unsuitable stabilization strategy (Camana et al 1977). Stability is theoretically improved by coupling the feedback, that is, making the motor commands for a single joint dependent on feedback from throughout the body (He et al 1991, Kuo 1995). Indeed, human postural behaviors appear to use heterogenic feedback, both during quiet standing (Speers et al 2002) and in response to large perturbations (Barin 1989, Park et al 2004). This behavior is equivalent to full state feedback, where each motor command is dependent on the positions and velocities of many-body segments, with closed-loop stability determined by body dynamics and a matrix of feedback gains.

The most simplistic implementation of this control is to feed sensory data (figure 1(b)) directly into a matrix of gains with no prior processing. The large variety and number of sensors is certainly sufficient to provide state information
(He et al 1991) needed for such direct feedback control (figure 1(c)). Where multiple sensors provide redundant data, the gain matrix effectively performs a weighted sum that will average out errors from noisy measurements. However, direct feedback does not adjust for sensory dynamics. For example, the semicircular canals act as high-pass filters of angular head velocity, yet posture control does not appear to be similarly filtered. Direct feedback is also not robust to sensory conflict. If one sensor conflicts with all others, it will nonetheless contribute to a compensatory motor command in direct proportion to its relative weight or gain within the feedback matrix. If sensory data were applied directly in feedback, posture control would be expected to exhibit the dynamics of the sensors, and to be highly sensitive to erroneous sensory inputs.

These drawbacks could be addressed by processing or filtering data as part of sensory integration. Such a processing 
system would receive sensory data as input, and produce the body state as output. An obvious method of removing sensory dynamics would be to invert each sensory transfer function. However, an inverse transfer function is not necessarily stable, for example the inverse of the semicircular canal's (high-pass filter) transfer function is unstable. The preferred means of processing sensory data is the Kalman filter, or optimal state estimator (figure 1(d)). It uses an internal, forward model of body and sensory dynamics to predict sensory output. The error in this prediction is then fed back through a set of estimator gains to correct the state estimate, which is in turn used for feedback control. A state estimator is a near-inverse of sensory dynamics that is both stable and causal. 'Optimal' refers to the design of estimator gains to minimize the mean-square estimation error, dependent on the presence of noise acting on the system. State estimators are used in modern control systems such as aircraft avionics and navigation systems, and have long been proposed as models for CNS sensory processing (Borah et al 1988, He et al 1991, Kuo 1995, Merfeld et al 1993, Wiener 1948).

We propose a computational model for posture control that combines state feedback and estimation. (Portions of this work were previously described in abbreviated form, Kuo (1997)). The state estimator processes information from multiple physiological sensors to provide the state information required for feedback control. Our model is stochastic, with noise acting as disturbances to the body and errors in sensory transduction. These noise characteristics are not easily measurable, and therefore serve as free parameters in the model. We examine the sensitivity of predicted posture behaviors to variations in these free parameters, and compare the stabilizing behaviors to human subjects from two age groups and one sensory loss group. Section 2 provides a brief background on models for human body and sensor dynamics, human perception, state feedback control and experimental methods. Section 3 describes the model, which integrates many of these components and is used to make predictions of observable behavior. Section 4 presents a comparison of theoretical predictions and experimental results along with a sensitivity analysis. Section 5 discusses biological and rehabilitative implications. Finally, section 6 summarizes the findings.

\section{Background}

Human posture is controlled with feedback from a variety of sensory organs. Three groups of sensors are thought to have primary responsibility in posture control: somatosensors, vestibular organs and vision (Horak and Macpherson 1995). The somatosensors are responsible for proprioception-the sensing of joint motion and limb position. Proprioception is dominated by muscle spindles, which are embedded within muscle and sense a combination of muscle length change and the rate of change. There are two types of vestibular organs. Semicircular canals are fluid-filled canals in the inner ear which detect angular velocity of the head by sensing viscous motion of the fluid; otoliths are crystal-like masses mounted on hair cells, and act as linear accelerometers. Vision is similarly sensitive to self-motion of the head. In the case of posture, the relevant signals are processed by motion detection circuitry not only in the retina but also in the visual cortex, so that the vestibular nuclei receive signals proportional to rotational and translational motion of the visual field (Young 1981).

These sensory signals are fed back to a series of hierarchical feedback loops to generate stabilizing motor commands. The conduit for all movement signals is the spinal cord (Horak and Macpherson 1995), which also produces the lowest level of neural feedback (see figure 1(a)). This feedback is in the form of local reflexes, in which stretch signals from a muscle are relayed to the spinal cord, passed across one or a few intermediate neural connections, and then fed directly back to the muscle, commanding a compensatory contraction. This short feedback loop has fast latency, in the range of 30-60 ms. However, the speed of such a loop comes with a disadvantage: local reflexes are the least integrative of postural responses, and are limited to relatively simple behaviors (Nashner 1977).

The second and most important level of feedback for balance control involves signals traveling up to the midbrain (see figure 1(a)). The brainstem serves as a relay and integration center, receiving and sending vast numbers of sensory and motor command signals. The mid-level feedback loop involves longer conduction paths and greater numbers of neural synapses, and consequently has a longer latency than spinal reflexes (often $90 \mathrm{~ms}$ and greater). But the convergence of many signals and complexity of connections allows the brain stem to generate much more complex movements, mostly of an automatic nature. The brainstem modulates the behavior of lower level reflexes, and is itself modulated by the next higher and final level. The cerebral cortex and related structures generate highly complex movements, mostly of a voluntary nature, with longer latencies than the two lower feedback loops (see figure 1). The longer latencies suggest that the cerebral cortex has a modulatory, rather than direct role in posture control. Brainstem lesions cause movement disorders affecting control of balance, indicating a more direct role for the mid-brain level of feedback (Nashner 1977).

Sensory integration can be assessed experimentally with clinical tests. A number of clinical tests exist for evaluating balance in patients suffering from instability (Baloh et al 1995). While some tests examine individual components such as a single sensory modality, others consider integrative, systems-level performance. The integrative tests involve applying perturbations to a movable support surface and recording the postural response, or recording motion or forces under the feet during quiet standing. One particularly integrative clinical test is the sensory organization test (SOT), also known as dynamic posturography (Mirka and Black 1990, Peterka and Black 1990), which uses a support surface which can be rotated about an axis approximately aligned to the ankles, and a visual surround which provides a visual field which can also be rotated about the same axis (see figure 2). In response to fore-aft body motion, the support surface and visual surround may be servo-driven (swayreferenced) to match the motion of the ankles. Swayreferencing of the support surface has the effect of rendering ankle proprioception inaccurate. Visual sway-referencing 


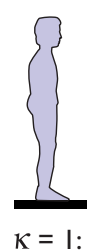

Normal

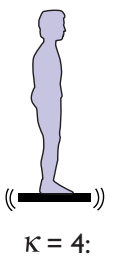

Platform

sway-ref

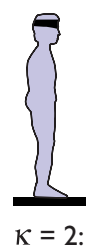

Eyes closed

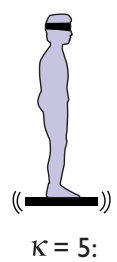

Eyes closed

Platform sway-ref

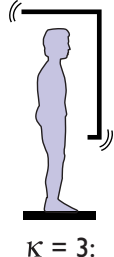

Vision sway-ref

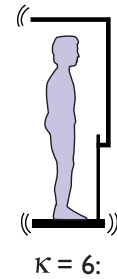

Vision sway-ref Platform sway-ref

Figure 2. Experimental apparatus for testing balance using dynamic posturography, or sensory organization test (SOT). Subject stands on a platform which can rotate about an axis collinear with the ankles. A visual surround can also rotate about this axis. Subjects can stand with platform and visual surround kept fixed, or with either servo-controlled to match lower body motion (using ankle angle as a reference). These are referred to as vision

sway-referenced and platform sway-referenced conditions, where visual or ankle proprioceptive feedback is rendered inaccurate. Combinations of these conditions (also with eye closure) test ability to integrate sensory data under demanding situations. The sensory condition number is referred to with the variable $\kappa=1,2, \ldots, 6$.

diminishes accuracy of visual sensing. Combinations of normal and sway-referenced vision and eyes closed (summarized in table 1), along with a fixed and swayreferenced support surface, are employed to test human response to altered sensory conditions. Subjects with absent vestibular function are unable to balance when platform swayreferencing is combined with either eyes closed or vision swayreferencing. However, the SOT appears to be less sensitive in diagnosing more subtle balance deficits, and test results offer limited insight to the cause of instability (Mirka and Black 1990). It is possible that models of balance control may aid interpretation of results. The present analysis models a modified version of the standard SOT (Speers et al 1999), in which ankle and hip joint kinematics are sensed directly rather than inferred from force plate measurements, as is the convention.

\section{Model of state estimation and control of balance}

The proposed model is based on several distinct components which are assembled into a single state space system. It makes use of a simplified model of human body dynamics, along with linear models of three sensory modalities: proprioception, vestibular organs and vision. The system combining these models is then controlled using state feedback, designed with linear quadratic regulator optimal control. A parametrization scheme makes it possible to describe the objective function with only two parameters and yet describe a wide range of postural behaviors. The state information for the resulting feedback is provided by a state estimator, whose behavior is determined by parameters describing sensor precision and other noise characteristics. These estimation parameters are difficult to determine independently, but can be constrained through observation of experimental behaviors. The overall system can then be altered to model the effects of visual and platform sway-referencing.

\subsection{Body dynamics model}

Human body dynamics are modeled as a two-segment inverted pendulum. Segment lengths and inertial properties are chosen to approximate those of a typical human (Kuo and Zajac 1993a). The two degrees of freedom correspond to motion about the ankles and hips in the sagittal plane (no sideto-side motion, and relatively small knee motion). The state-space representation will use states based on segment coordinates $\phi_{1}$ and $\phi_{2}$, defined as the angle of the lower leg and trunk measured counter-clockwise with respect to the vertical, respectively. This model assumes that lowerlevel circuits translate commands coding desired movement into motor signals to the muscles. The command inputs may therefore be expressed in terms of two-dimensional joint motion, rather than the higher-dimensional motor signals. However, constraints on muscle force are still incorporated indirectly by calculating the set of feasible accelerations of a model including fourteen muscle groups (Kuo and Zajac 1993a, 1993b), and then performing a reduced-order approximation to that set (Kuo 1995). The result is a simpler system that still models the mass and inertial coupling of the body. Its state equations are in standard form,

$$
\dot{x}_{\mathrm{B}}=A_{\mathrm{B}} x_{\mathrm{B}}+B_{\mathrm{B}} u_{\text {control }}
$$

where the state is defined as $x_{\mathrm{B}} \triangleq\left[\begin{array}{llll}\phi_{1} & \phi_{2} & \dot{\phi}_{1} & \dot{\phi}_{2}\end{array}\right]^{\mathrm{T}}$, and the subscript ' $\mathrm{B}$ ' denotes the body. The system matrices are

$$
A_{\mathrm{B}} \triangleq\left[\begin{array}{cc}
0 & I^{2 \times 2} \\
G & 0
\end{array}\right], \quad B_{\mathrm{B}} \triangleq\left[\begin{array}{c}
0 \\
H
\end{array}\right],
$$

where $G$ and $H$ are matrices summarizing the inertial properties (see the appendix for numerical values), $I^{2 \times 2}$ is the $2 \times 2$ identity matrix, and $u_{\text {control }}$ corresponds to two directions of motion, scaled to equal command effort.

\subsection{Sensor models}

The following sensors are to be modeled: ankle and hip proprioception, visual rotation and translation, and vestibular rotation and translation. For each type of sensor, a simple linear model is employed, presented in transfer function form for simplicity. For interfacing with body dynamics, however, each transfer function is realized in a state-space form,

$$
\begin{aligned}
& \dot{x}_{s}=A_{s} x_{s}+B_{s} u_{s} \\
& y_{s}=C_{s} x_{s}+D_{s} u_{s}
\end{aligned}
$$

where $y_{s}$ is the sensory output, $u_{s}$ the stimulus input, $x_{s}$ the sensor states, and ' $s$ ' is replaced by a specific subscript for each sensor. System matrices $\left\{A_{s}, B_{s}, C_{s}, D_{s}\right\}$ denote a state-space 
realization equivalent to that sensor's transfer function. The following two-letter subscripts will be used to refer to each sensory modality: 'ap' for ankle proprioception, 'hp' for hip proprioception, 'sc' for semi-circular canals, 'ot' for otoliths, 'vr' for visual rotation, and ' $v t$ ' for visual translation.

Muscle spindles are modeled as simple lead-lag filters which provide a signal proportional to length and velocity within a limited bandwidth. The transfer function from length change to spindle output is (Agarwal and Gottlieb 1984)

$$
\frac{Y_{\text {spindle }}}{X_{\text {length }}}=\frac{T_{\mathrm{sp}} s+1}{\alpha T_{\mathrm{sp}} s+1},
$$

where $\alpha$ and $T_{\text {sp }}$ are lead-lag constants (see the appendix for numerical values). Two sets of muscle spindles are included, at the ankles and at the hips. The muscle moment arm about these joints is assumed to be nearly constant around the operating point, adding a proportionality factor which is normalized to one. A state-space realization of equation (3) for each proprioceptor is given by the $(1 \times 1)$ matrices

$$
\begin{array}{ll}
A_{\mathrm{ap}} \triangleq A_{\mathrm{hp}} \triangleq \frac{-1}{\alpha T_{\mathrm{sp}}}, & B_{\mathrm{ap}} \triangleq B_{\mathrm{hp}} \triangleq \frac{1}{\alpha T_{\mathrm{sp}}}, \\
C_{\mathrm{ap}} \triangleq C_{\mathrm{hp}} \triangleq \frac{\alpha-1}{\alpha}, & D_{\mathrm{ap}} \triangleq D_{\mathrm{hp}} \triangleq \frac{1}{\alpha},
\end{array}
$$

with the inputs to the sensors set to the ankle and hip joint angles,

$$
u_{\mathrm{ap}}=\phi_{1}, \quad u_{\mathrm{hp}}=\left(\phi_{2}-\phi_{1}\right) .
$$

Each realization may be verified to be equivalent to the transfer function of equation (3), through the transformation

$$
\frac{Y_{s}}{U_{s}}=C_{s}\left(s I-A_{s}\right)^{-1} B_{s}+D_{s}
$$

where $I$ is the identity matrix.

Semicircular canals have an overdamped response to angular acceleration, which is detected by hair cells whose firing rate is roughly proportional to angular velocity. Fernandez and Goldberg (1971) proposed a linear model with transfer function from head angular acceleration to neural firing rate. We modified their model to include a limitation on bandwidth, both to constraint high-frequency response and to ensure the overall system's causality:

$$
\frac{Y_{\mathrm{sc}}}{X_{\text {angacc }}}=\frac{k_{\mathrm{sc}} s\left(s+\omega_{s 1}\right)}{\left(s+\omega_{s 2}\right)\left(s+\omega_{s 3}\right)},
$$

where $k_{\mathrm{sc}}, \omega_{s 1}, \omega_{s 2}$ and $\omega_{s 3}$ are constant parameters (see the appendix for numerical values of constants). The canals are arranged in pairs oriented in mutually orthogonal directions, none of which are aligned with head rotation in the sagittal plane. However, because vestibular processing has been demonstrated to perform coordinate transformations (Merfeld et al 1993), a single pair of canals is incorporated in the present model, with state-space matrices $\left\{A_{\mathrm{sc}}, B_{\mathrm{sc}}, C_{\mathrm{sc}}, D_{\mathrm{sc}}\right\}$ and input

$$
u_{\mathrm{sc}}=\ddot{\phi}_{2} \text {. }
$$

The otoliths respond to translational acceleration by detecting deflection of hair cell mounted with massive crystals. Young and Meiry (1968) found a linear transfer function

$$
\frac{Y_{\mathrm{ot}}}{X_{\text {linacc }}}=\frac{k_{\mathrm{ot}}\left(s+\omega_{o 1}\right)}{\left(s+\omega_{o 2}\right)}
$$

where $k_{\mathrm{ot}}, \omega_{o 1}$ and $\omega_{o 2}$ are constant parameters (see the appendix for numerical values of constants). Though otoliths are found in two locations, the utricle and saccule, on each side of the head, a single pair is modeled here, detecting only fore-aft linear translation of the head, which is in turn fixed rigidly to the trunk. The corresponding state-space matrices are $\left\{A_{\mathrm{ot}}, B_{\mathrm{ot}}, C_{\mathrm{ot}}, D_{\mathrm{ot}}\right\}$ with input

$$
u_{\mathrm{ot}}=c_{t 1} \ddot{\phi}_{1}+c_{t 2} \ddot{\phi}_{2}
$$

where $c_{t}^{\mathrm{T}} \triangleq\left[\begin{array}{ll}c_{t 1} & c_{t 2}\end{array}\right]$ transforms the angular accelerations into linear translation of the head (see the appendix for numerical values of constants).

The eyes respond to both visual rotation and translation (Robinson 1981). Many of the cues contributing to selfmotion come from peripheral vision, and much of the signal processing necessary to detect motion is performed in the retinal circuitry. Further processing is performed by ganglion cells and the visual cortex, with a large number of connections contributing to significant phase lag. A simplified model for visual signal processing includes detection of velocity of sagittal plane rotation and of fore-aft translation, with a bandwidth limitation:

$$
\frac{Y_{\mathrm{v}}}{X_{\mathrm{v}}}=\frac{1}{T_{v} s+1} .
$$

$T_{v}=0.1 \mathrm{~s}$ is based on visual processing lag (Robinson 1981). The phase lag for this transfer function is likely more of more behavioral significance than the magnitude. The velocity input for rotation and translation is head (fixed to trunk) angular velocity and head fore-aft translational velocity, with resulting state space matrices $\left\{A_{\mathrm{vr}}, B_{\mathrm{vr}}, C_{\mathrm{vr}}, D_{\mathrm{vr}}\right\}$ and $\left\{A_{\mathrm{vt}}, B_{\mathrm{vt}}, C_{\mathrm{vt}}, D_{\mathrm{vt}}\right\}$ and inputs

$$
u_{\mathrm{vr}}=\dot{\phi}_{2}, \quad u_{\mathrm{vt}}=c_{t 1} \dot{\phi}_{1}+c_{t 2} \dot{\phi}_{2} .
$$

The individual component models are assembled into a comprehensive system, describing the combined dynamics of the body and sensors. Body and sensor dynamics (equations (1), (4), (5), (7), (9), (11) and (12)) together form the system matrices $A_{\mathrm{BS}}, B_{\mathrm{BS}}, C_{\mathrm{BS}}$ and $D_{\mathrm{BS}}$, where the subscript 'BS' refers to the body and sensors, and the state vector is defined as $x_{\mathrm{BS}}^{\mathrm{T}} \triangleq\left[\begin{array}{lllllll}x_{\mathrm{B}}^{\mathrm{T}} & x_{\mathrm{ap}}^{\mathrm{T}} & x_{\mathrm{hp}}^{\mathrm{T}} & x_{\mathrm{sc}}^{\mathrm{T}} & x_{\mathrm{ot}}^{\mathrm{T}} & x_{\mathrm{vr}}^{\mathrm{T}} & x_{\mathrm{vt}}^{\mathrm{T}}\end{array}\right]$. This system is subject to process noise $w$, and the sensors to measurement noise $v$, both modeled as zero-mean Gaussian white-noise processes. Process noise encompasses unmodeled dynamics, random external perturbations to equilibrium, and internal effects such as random fluctuations in muscle force, all acting at relative levels similar to that of $u_{\text {control }}$. Because the body dynamics act as a dominant low-pass filter, it is unnecessary to impose a bandwidth limitation on the noise inputs. Measurement noise describes the signal-to-noise ratios of sensory signals, which depend on such effects as precision of sensors, loss of fidelity due to synapses and transmission distance, and general information capacity in the sensory system. Together, these additions result in state equations

$$
\begin{gathered}
\dot{x}_{\mathrm{BS}}=A_{\mathrm{BS}} x_{\mathrm{BS}}+B_{\mathrm{BS}} u_{\text {control }}+B_{\mathrm{BS}} w \\
y_{s}=C_{\mathrm{BS}} x_{\mathrm{BS}}+D_{\mathrm{BS}} u_{\text {control }}+v .
\end{gathered}
$$

The noise characteristics of $w$ and $v$ constitute the unknown parameters of the model. 


\subsection{State feedback control}

State feedback gains for stabilizing the body are determined by a parametrized objective function for linear quadratic optimal control (Kuo 1995). Parametrization is helpful for varying the shape of the state objective, in accordance with changes in behavior such as scaling of control with perturbation magnitude (Park et al 2004). The state feedback gains for posture may generally differ from those produced by optimal control, but linear quadratic control provides a means to use relatively few parameters to describe a large number of gains, and it guarantees a large degree of robustness to time delays (Kuo 1995). The quadratic objective function is

$$
J=\int_{0}^{\infty}\left(x_{\mathrm{B}}^{\mathrm{T}} Q x_{\mathrm{B}}+u_{\text {control }}^{\mathrm{T}} R u_{\text {control }}\right) \mathrm{d} t
$$

The control weighting matrix $R$ is set to the identity matrix, because the entries of $u_{\text {control }}$ are proportional to actual neural command effort. Weighting of the state objective relative to control effort is set by a single parameter, $\sigma$. The shape of the ankle versus hip trajectory is set by another parameter, $\mu$ (described in detail in Kuo (1995)). The two parameters define $Q$ with the equation

$$
Q=\sigma^{2} \frac{\mu Q_{\mathrm{cm}}+(1-\mu) Q_{\mathrm{up}}}{\lambda_{\max }\left(\mu Q_{\mathrm{cm}}+(1-\mu) Q_{\mathrm{up}}\right)}
$$

where the function $\lambda_{\max }(A)$ refers to the maximum eigenvalue of a matrix $A$. Parameter $\mu$ sets the joint trajectory shape by varying the proportion of two objectives $Q_{\mathrm{up}}$ and $Q_{\mathrm{cm}}$. $Q_{\text {up }}$ weights joint angles equally, reflecting an unbiased desire to maintain upright equilibrium. $Q_{\mathrm{cm}}$ is a penalty objective modeling the relative undesirability of allowing the body center of mass to approach the limits of the base of support (see the appendix for numerical values). The relative proportion of this penalty increases with perturbation size, resulting in a continuum of behaviors approximating those seen experimentally (Kuo 1995). Equation (13) can be minimized with feedback control, determined by solving the algebraic Riccati equation (Bryson 2002),

$$
\begin{gathered}
A_{\mathrm{B}}^{\mathrm{T}} S+S A_{\mathrm{B}}-S B_{\mathrm{B}} R^{-1} B_{\mathrm{B}}^{\mathrm{T}} S+Q=0 \\
K_{\mathrm{B}}=R^{-1} B_{\mathrm{B}}^{\mathrm{T}} S .
\end{gathered}
$$

These equations are solved for $K_{\mathrm{B}}$, the matrix describing the gains of the states to the control, and $S$, an intermediate quantity (sometimes referred to as the 'cost-to-go matrix'). This feedback is applied to the entire system (12) using the augmented matrix

$$
K_{\mathrm{BS}}=\left[\begin{array}{ll}
K_{\mathrm{B}} & 0
\end{array}\right]
$$

with the feedback control given by

$$
u_{\text {control }}=-K_{\mathrm{BS}} x_{\mathrm{BS}} .
$$

\subsection{State estimator model}

CNS sensory processing is modeled by an optimal state estimator, which estimates the full state $x_{\mathrm{BS}}$ of the body and sensors for use in the feedback control. The estimate is produced by an internal model of the body and sensory dynamics (figure 1(d)). This model can produce both a state estimate $\hat{x}_{\mathrm{BS}}$ and a prediction $\hat{y}_{s}$ of sensory output. Process noise and other disturbances can cause the actual state to deviate from this estimate. This deviation can be corrected with feedback of the sensory prediction error $y_{s}-\hat{y}_{s}$, with estimator gain matrix $L$ determining the correction's effect on each state:

$$
\begin{gathered}
\dot{\hat{x}}_{\mathrm{BS}}=A_{\mathrm{BS}} \hat{x}_{\mathrm{BS}}+B_{\mathrm{BS}} u_{\text {control }}+L\left(y_{s}-\hat{y}_{s}\right) \\
\hat{y}_{s}=C_{\mathrm{BS}} \hat{x}_{\mathrm{BS}}+D_{\mathrm{BS}} u_{\text {control }} .
\end{gathered}
$$

The principal problem in state estimation is the design of the estimator gains $L$ that govern the correction of estimate $\hat{x}_{\mathrm{BS}}$ based on sensory feedback $y$. There are many criteria by which $L$ may be designed, with the most important requirement being the stabilization of the estimation error $x_{\mathrm{BS}}-\hat{x}_{\mathrm{BS}}$. We employ optimal state estimation, which minimizes the mean-square error for a particular set of process and sensor noise characteristics. One advantage of optimal state estimation is that a relatively large number of entries in $L$ may effectively be parametrized by a smaller number of noise power spectral densities. Another advantage is that minimization of mean-square error could potentially be the outcome of an adaptation process mediated by the cerebellum (Paulin 1989). Optimization might therefore be a physiological explanation for how humans might learn to perform state estimation by iteratively adjusting feedback gains to minimize prediction error. In the postural control model, estimator gains $L$ are computed using the algebraic Riccati equation:

$$
\begin{gathered}
\tilde{X} A_{\mathrm{BS}}^{\mathrm{T}}+A_{\mathrm{BS}} \tilde{X}-\tilde{X} C_{\mathrm{BS}}^{\mathrm{T}} V^{-1} C_{\mathrm{BS}} \tilde{X}+W=0 \\
L=\tilde{X} C_{\mathrm{BS}} V^{-1},
\end{gathered}
$$

where $W$ and $V$ are diagonal matrices containing the power spectral densities for $w$ and $v$, respectively, and $\tilde{X}$ is the steady-state estimator error covariance. These equations are solved for $\tilde{X}$ and $L$. With both $w$ and $v$ assumed to be white and independent with zero mean, power spectral densities are sufficient to fully describe the process and sensor noise. Process noise is assumed to be of equal magnitude for each input, so that $W$ is set by a single parameter $W_{1}$ :

$$
W \triangleq\left[\begin{array}{ll}
W_{1} & \\
& W_{1}
\end{array}\right] .
$$

$V$ is parametrized by a set of noise-to-signal power ratios $\pi_{\mathrm{ap}}, \pi_{\mathrm{hp}}, \ldots$ (Borah et al 1988). These ratios describe power spectral densities relative to the output covariance $Y$ of the state feedback-stabilized system driven by process noise alone,

$$
Y=C_{\mathrm{BS}} X_{\mathrm{fb}} C_{\mathrm{BS}},
$$

where $X_{\mathrm{fb}}$ is the state covariance, found by solving the equation $\left(A_{\mathrm{BS}}-B_{\mathrm{BS}} K_{\mathrm{BS}}\right) X_{\mathrm{fb}}+X_{\mathrm{fb}}\left(A_{\mathrm{BS}}-B_{\mathrm{BS}} K_{\mathrm{BS}}\right)^{\mathrm{T}}+B_{\mathrm{BS}} W B_{\mathrm{BS}}^{\mathrm{T}}=0$. 
The sensor noise power spectral densities are then defined as

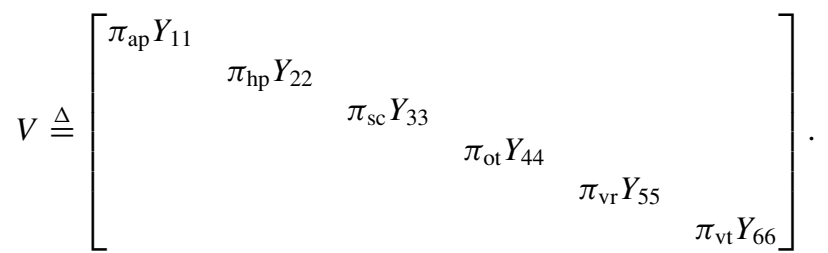

The state feedback and estimator are combined into a linear quadratic Gaussian (LQG) system. The parameters that determine the behavior of this system are

$$
\pi^{\mathrm{T}} \triangleq\left[\begin{array}{lllllllll}
\sigma & \mu & W_{1} & \pi_{\mathrm{ap}} & \pi_{\mathrm{hp}} & \pi_{\mathrm{sc}} & \pi_{\mathrm{ot}} & \pi_{\mathrm{vr}} & \pi_{\mathrm{vt}}
\end{array}\right],
$$

so that two parameters describe the control feedback, and the remaining seven parameters describe process and sensor noise. The stochastic nature of this system also means that the closed-loop system is not designed to predict explicit joint trajectories. Rather, the model is used to predict the covariance of joint motion, $X_{\mathrm{LQG}}$ about upright equilibrium. Estimator state covariance $\hat{X}$ is found by solving the equation

$\left(A_{\mathrm{BS}}-B_{\mathrm{BS}} K_{\mathrm{BS}}\right) \hat{X}+\hat{X}\left(A_{\mathrm{BS}}-B_{\mathrm{BS}} K_{\mathrm{BS}}\right)^{\mathrm{T}}+L V L^{\mathrm{T}}=0$

and $X_{\mathrm{LQG}}$ is the sum of estimator state and error covariances,

$$
X_{\mathrm{LQG}}=\hat{X}+X_{e} \text {. }
$$

Of these covariances, only those corresponding to $x_{\text {body }}$ can be measured experimentally, and the preferred coordinate system for comparisons is

$$
x_{\theta} \equiv\left[\begin{array}{c}
\theta_{\text {shk }} \\
\theta_{\text {hip }}
\end{array}\right]=c_{\theta} x_{\mathrm{BS}}=\left[\begin{array}{ccccc}
-1 & 1 & 0 & \cdots & 0 \\
0 & 1 & 0 & \cdots & 0
\end{array}\right] x_{\mathrm{BS}}
$$

where $\theta_{\text {shk }}$ and $\theta_{\text {hip }}$ are the shank and hip angles, respectively (Kuo et al 1998). The shank angle is defined as the angle of the shank relative to the support surface, and hip angle as the angle of the trunk with respect to the leg (see figure 3), both increasing in the extension direction. The corresponding covariance is

$$
X_{\mathrm{LQG} \theta}=c_{\theta}^{\mathrm{T}} X_{\mathrm{LQG}} c_{\theta} \text {. }
$$

\subsection{Models of sensory conditions}

Altered sensory conditions imposed by the sensory organization test affect the behavior of the system significantly, and are modeled by making changes to the nominal state equations. These changes may also be accompanied by changes in the state estimator gains, depending on the subject's awareness of those changes. We assume that when subjects consciously close their eyes, they are aware that visual information is no longer available, and may make adjustments to how the remaining sensory inputs are weighted. We model this as a new set of state estimator gains $L$. However, in the sway-referencing conditions, we assume that subjects are unaware that sensory information has been rendered inaccurate, and use the nominal set of state estimator gains. We therefore model the inaccuracy induced by sway-referencing with appropriate changes to the state equations, but with no changes to the estimator gains $L$.

The eyes closed condition is modeled by removing the visual states $x_{\mathrm{vr}}$ and $x_{\mathrm{vt}}$, and their associated outputs. This is

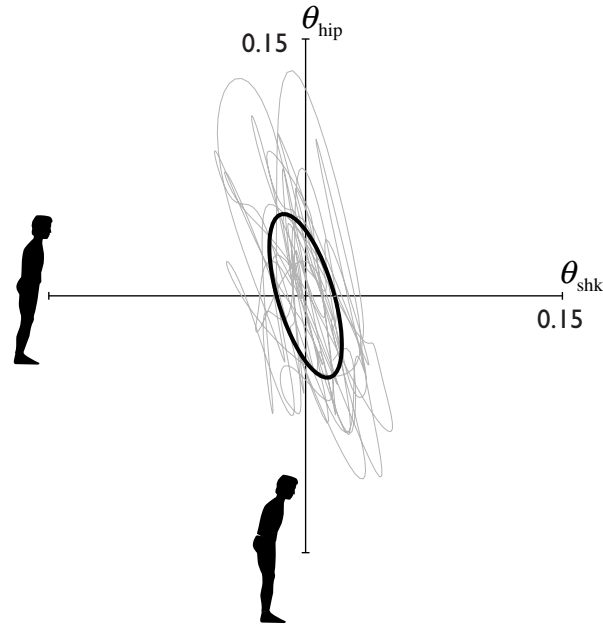

Figure 3. Covariance ellipse summarizes amount of sway about ankles and hips during upright standing. Motion of the body is measured in terms of angle of the shank with respect to support surface normal, $\theta_{\text {shk }}$, and motion of the trunk with respect to the shank, $\theta_{\text {hip }}$. A typical trace of $\theta_{\text {hip }}$ versus $\theta_{\text {shk }}$ is shown during quiet standing, under normal sensory conditions. The covariance matrix describes the variances in the hip and shank individually, along with the coupling between the two joints. A single ellipse, representing one standard deviation of sway, summarizes the entire covariance.

accomplished by the equivalent action of setting the noise-tosignal parameters $\pi_{\mathrm{vr}}$ and $\pi_{\mathrm{vt}}$ to infinity, and then re-computing the state estimation gains accordingly. Closing the eyes is the only condition in which the subject is explicitly aware of a change in sensory conditions, and so the estimator gains are recalculated taking this into account.

Visual sway-referencing takes place without the subject's awareness, with the assumption that the CNS does not alter its control behavior under this condition. However, the eyes receive inaccurate motion cues, modeled as separate effects on visual rotation and translation. Ankle-driven swayreferencing only partially affects visual rotation cues, because the hips induce significant motions of the upper body that are not countered by the sway-referencing servo controller. In contrast, visual translation cues are affected to a larger degree because translation of the head is dominated more by (inaccurate) ankle motion than by hip motion (see figure 2). The visual translation sensory input is therefore modeled as having zero gain, whereas the visual rotation input is modeled as feedback of hip motion alone, with the ankle-driven motion of the visual surround removed. For this condition, equations (11) are replaced by

$$
u_{\mathrm{vr}}=\dot{\phi}_{2}-\dot{\phi}_{1}, \quad u_{\mathrm{vt}}=0 .
$$

Lack of subject awareness is modeled by retaining the original state estimator for the intact system, so that the weightings of visual rotation and translation are unchanged. The state estimate therefore depends on the inaccurate information to the same degree as if the visual inputs were accurate.

Platform sway-referencing also takes place without the subject's awareness, and is modeled by a feedback control performed on the support surface under the feet. Motion of the support surface, $\ddot{\phi}_{\mathrm{p}}$, not only acts as an inaccurate reference 
for ankle proprioception, but also acts as a small disturbance to the body. The platform dynamics are modeled as a servodriven second-order system,

$$
\ddot{\phi}_{\mathrm{p}}=-k_{\mathrm{p}}\left(\phi_{\mathrm{p}}-\phi_{1}\right)-b_{\mathrm{p}} \dot{\phi}_{\mathrm{p}},
$$

with gains $k_{\mathrm{p}}=400 \mathrm{~s}^{-2}$ and $b_{\mathrm{p}}=32 \mathrm{~s}^{-1}$ chosen to make the platform much faster than the body dynamics. These dynamics are augmented to the regular state equations (12), and the disturbance effect is computed by deriving the body dynamics including an extra degree of freedom for the platform. Under platform sway-referencing, equation (1) is altered to include this disturbance:

$$
\dot{x}_{\mathrm{B}}=A_{\mathrm{B}} x_{\mathrm{B}}+B_{\mathrm{B}} u_{\text {control }}+B_{\mathrm{p}} \ddot{\phi}_{\mathrm{p}}
$$

where $B_{\mathrm{p}}$ describes the effect of platform acceleration on the body, derived from the dynamical equations of motion. As with visual sway-referencing, platform sway-referencing only affects the body dynamics, feeding back inaccurate information to an estimator that was designed for the original, intact system.

These sensory conditions make the overall behavior of the closed-loop system a function of not only the nominal parameters $\pi$ but also the sensory condition number $\kappa$. We denote this function $X_{\mathrm{LQG}} \theta(\kappa ; \pi)$, specifically excluding independent anthropometric and sensory parameters that remain relatively fixed. There are nine degrees of freedom in the free parameters $\pi$, of which two-the state feedback parameters $\sigma$ and $\mu$-are determined by separate perturbation experiments. The remaining parameters in $\pi$ describe noise characteristics and are therefore determined from experimental observations of stochastic upright balancing.

\section{Comparisons with experimental data}

The model was tested by comparing theoretical joint angle covariances under altered sensory conditions against those measured experimentally. Some unknown parameters of vector $\pi$ were necessarily calibrated using these data; the model was therefore not purely predictive. However, the large number of degrees of freedom in the possible observable behaviors, compared to the smaller number of free parameters, means that data could constrain the model to a considerable extent. The most meaningful tests were to determine whether the model successfully predicted trends in behavior as a function of sensory condition, and how sensitive this same model was to variations in parameter values.

\subsection{Comparison with healthy young adult subjects}

Experimental data were recorded from sensory organization tests performed on eight subjects, in order to compute empirical joint angle covariances using a protocol described previously (Kuo et al 1998). Subjects were young (age $<45$ years), healthy normal adults. One trial each of conditions 1 and 2 , and three trials of conditions 3 through 6 , were performed. Recorded data included shank and hip kinematics $\left(\theta_{\text {shk }}\right.$ and $\theta_{\text {hip }}$, see figure 3$)$ and ground reaction forces, all sampled at $50 \mathrm{~Hz}$. Trials lasted $21 \mathrm{~s}$, with $1 \mathrm{~s}$ at the beginning discarded to reduce transients. Data were band-pass filtered using a third-order digital Butterworth filter, with high-pass cutoff of $0.5 \mathrm{~Hz}$ and low-pass cutoff at $3.5 \mathrm{~Hz}$. The high-pass cutoff was used to filter out very slow drift, so that covariances were computed on at least 10 cycles of the lowest remaining frequency components. The low-pass cutoff was employed to filter out noise. Sway-referencing commands were based on feedback of the shank angle. For simplicity, sample covariances were displayed for only $\theta_{\text {shk }}$ and $\theta_{\text {hip }}$, forming a subset of the predicted covariance matrix without the angular velocities. Mean covariances across all subjects were used for $X_{\exp \theta}(\kappa)$. Details of the experimental procedure are given in Kuo et al (1998). A sample trace of $\theta_{\text {shk }}$ and $\theta_{\text {hip }}$, along with the covariance ellipse, is shown in figure 3 . The horizontal extent of the ellipse corresponds to $\theta_{\text {shk }}$ variance, the $(1,1)$ element of $X_{\exp \theta}(\kappa)$, and the vertical extent to $\theta_{\text {hip }}$ variance, the $(2,2)$ element of $X_{\exp \theta}(\kappa)$. Covariance of $\theta_{\text {shk }}$ and $\theta_{\text {hip }}$, the off-diagonal element, is distinguished by a tilt to the ellipse with respect to vertical.

The feedback control system parameters were the most straightforward to determine. The state objective parameter $\mu$ determines the relative contribution of hip motion to posture control, with a value near 1 for responding to large disturbances (Kuo 1995). In quiet standing, there is relatively little hip motion, with $\mu$ approaching zero. Because the sensory organization test performs no explicit perturbations and subjects perform quiet standing in all conditions, we set $\mu=0$. Process noise covariance $W_{1}$ was set to 0.08 and $\sigma$ was set to 2.5 in order to match the magnitudes of covariance for $\kappa=1$. These parameters were then kept constant for all comparisons.

The remaining parameters were associated with sensor noise-to-signal ratios. Manipulation of the sensor parameters resulted in a relatively narrow range of behaviors for some sensory conditions despite extreme variations in parameters. Indeed, such robustness enabled the determination of $\sigma, \mu$ and $W_{1}$ prior to setting the other parameter values. Conditions $\kappa=5$ and $\kappa=6$ were relatively the most sensitive to these remaining parameters, and were used as the basis for finding the following values, which produced a relatively good match to experimental data:

$$
\begin{gathered}
\pi_{\mathrm{ap}}=0.05, \quad \pi_{\mathrm{hp}}=0.01, \\
\pi_{\mathrm{sc}}=\pi_{\mathrm{ot}}=\pi_{\mathrm{vr}}=\pi_{\mathrm{vt}}=0.001 .
\end{gathered}
$$

These parameter values, along with the sway-referencing models, together predict a single set of sway covariances for all six SOT conditions (see figure 4). State estimation makes the system stable for all conditions despite removal or inaccuracy of some sensory information. For example, the model treats the eyes closed $(\kappa=2)$ condition as a reduction in sensory channels that results in slightly greater sway. Interestingly, the visual sway-referencing $(\kappa=3)$ condition has less effect on sway, because the model allows some useful motion cues to be detected in that condition. If visual motion could completely be removed, the model would predict a larger effect than for eyes closed. The model is also relatively unaffected by platform sway-referencing $(\kappa=4)$, indicating that sufficient information is available from other sensors to permit precise stabilization. However, when 
(a) State Estimator Model of Normal Posture

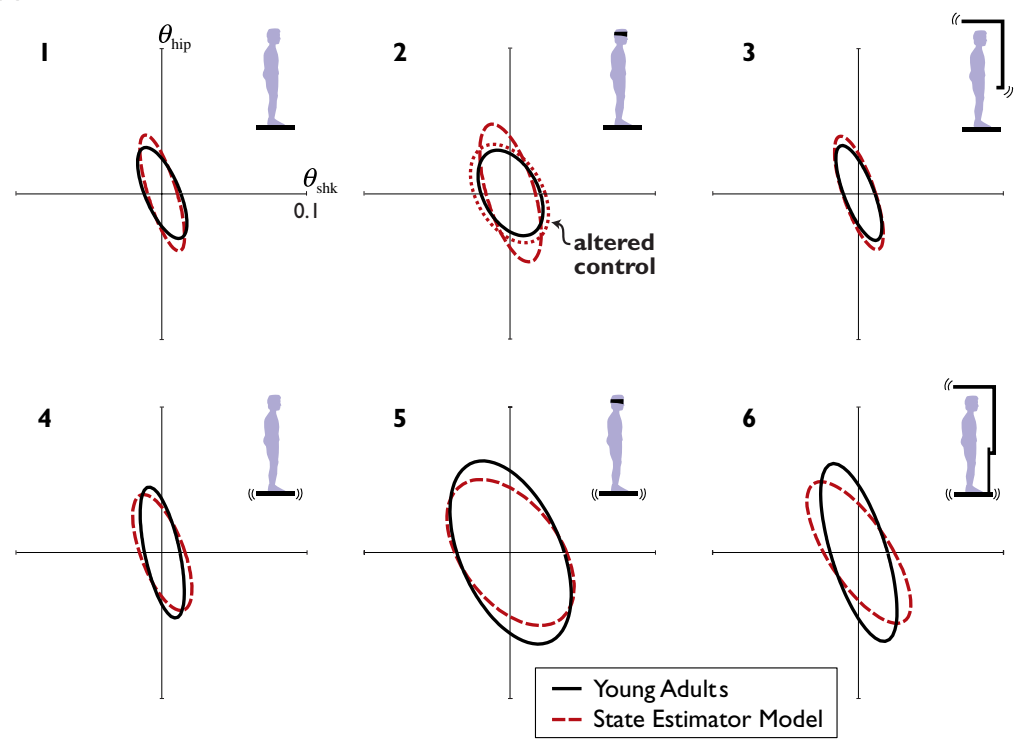

(b) Direct Feedback Model of Normal Posture

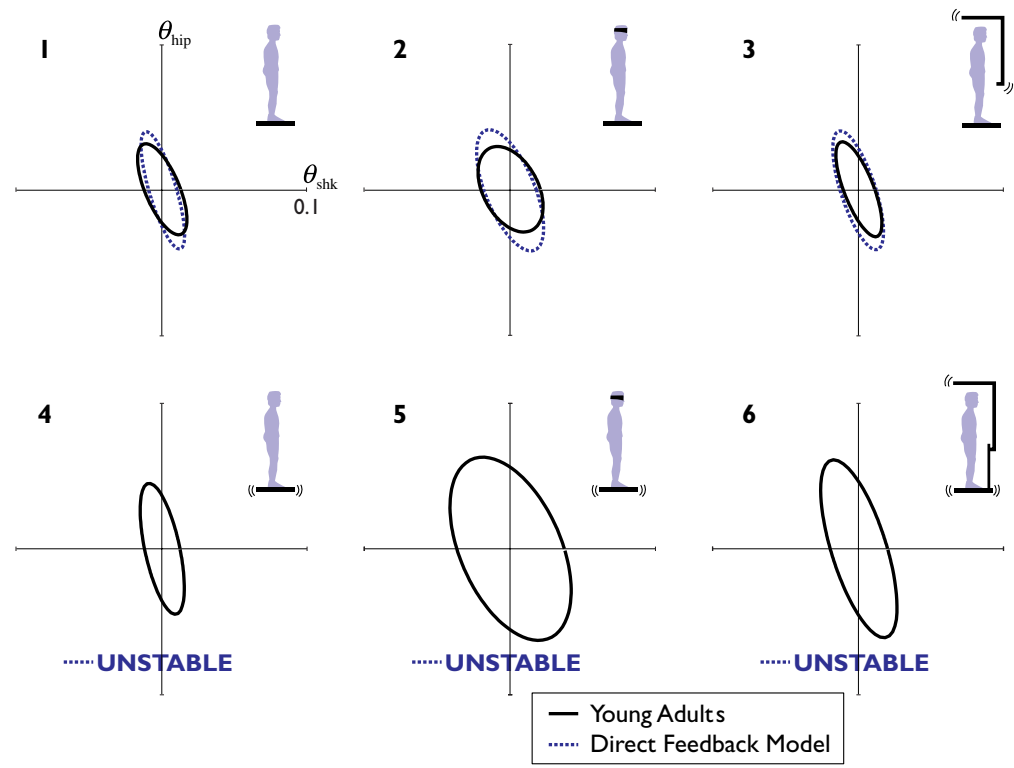

Figure 4. Comparison of experimental results from normal young adults $(N=8)$ against (a) state estimator and (b) direct feedback models, for six sensory conditions. Model covariance ellipses were computed for a consistent environment of process noise and measurement noise. The eyes closed condition $(\kappa=2)$ was modeled by removal of vision, and the vision sway-referenced condition $(\kappa=3)$ by reducing the feedback from visual translation and rotation, as if the visual surround were driven by ankle motion. Platform sway-referencing $(\kappa=4-6)$ was modeled by applying a servo command to the support surface, also driven by ankle motion. (a) State estimator model produces changes in covariance similar to normals as a function of sensory condition $\kappa$. Both model and experiment show small increases in sway in conditions where only one type of sensory feedback is affected $(\kappa=1-4)$, with the most sway when two types are affected $(\kappa=5,6)$. All parameter values are kept fixed when computing model covariances for different sensory conditions. In the eyes closed condition $(\kappa=2)$, human subjects sway less than might be expected from the loss of sensory information. This behavior could potentially be explained by a change in control strategy, modeled by increasing parameter $\sigma$ (denoted 'altered control', ellipse with dotted line). The resulting covariance is smaller and more similar to that observed experimentally. (b) Direct feedback model can also produce covariances similar to those observed experimentally, for three of the sensory conditions $(\kappa=1-3)$. However, for platform sway-referenced conditions $(\kappa=4-6)$ the dependence on inaccurate ankle feedback causes the direct feedback model to be unstable. In contrast, both normal humans and the state estimator model are remarkably robust to inaccurate sensory information.

inaccurate ankle proprioception is combined with eyes closed $(\kappa=5)$ or visual sway-referencing $(\kappa=6)$ conditions, the model predicts a much larger increase in sway. This is due to the fact that accurate information is received only through hip proprioception and the vestibular organs. Despite this reduction of sensory information, the model is able to robustly 
maintain stability, with the only penalty being an increase in sway.

These model's sway covariance ellipses compare well with those measured in healthy young adults (see figure 4(a)). The state estimator model produces behaviors that are qualitatively similar to how humans compensate for different sensory conditions. The model agrees with experimental data showing that covariance for $\kappa=3$, visual sway-referencing, is similar to that for $\kappa=1$. The data also show a relatively small increase in hip sway under platform sway-referencing $(\kappa=4)$, as compared to normal support surface $(\kappa=1)$. The largest disagreement between model and data was for eyes closed $(\kappa=2)$, where the model predicted approximately $50 \%$ higher hip sway than seen experimentally. This may be due to the fact that the model employs the same state feedback gains $K_{\mathrm{B}}$ regardless of sensory condition. Human subjects may, in contrast, employ higher gain when their eyes are closed. Indeed, this same behavior could easily be produced by increasing control parameter $\sigma$ for this condition (see 'altered control' in figure 4(a)).

\subsection{Comparison with direct feedback model}

An alternative to state estimation is for each sensory channel to be filtered independently, and for all such signals to be directly applied to feedback control without passing through an internal model (figure 1(c)). Direct feedback can include pathways for each sensory signal to contribute to control of each joint or muscle, through a matrix of feedback gains. However, each sensor's feedback is taken literally, rather than corroborated against an internal representation of the body's state. One of the arguments for state estimation is that such corroboration can make the posture control system more robust to inaccurate sensory information.

We tested the effect of altered sensory conditions on a model of direct feedback (see figure 4(b)). We used the same feedback control system as for the state estimation model, but assumed that sensory dynamics are inverted perfectly to yield direct measurements of the states. For ankle proprioception, the muscle spindle dynamics were assumed to be filtered to yield shank angle and velocity. These were then fed directly into the control gains $K$, without need for a state estimator. Under ideal conditions, the resulting system has identical performance to the state estimator-based control. However, under altered sensory conditions, direct feedback and estimator-based feedback predict very different behaviors.

Sway-referencing of the platform support surface $(\kappa=$ 4-6) was found to immediately destabilize the direct feedback system. With no feedback from other sensors to indicate ankle motion, the system cannot function with inaccurate ankle proprioception. In fact, the system becomes stable even for a 50\% reduction in ankle gain by platform sway-referencing. This is because direct feedback takes each sensory channel literally as an indicator of joint or limb position, so that platform sway-referencing has an effect equivalent to reducing the feedback gains associated with ankle position and velocity.

This high sensitivity to inaccurate sensing is in stark contrast to state estimation. To an internal representation of body and limb dynamics, ankle proprioception is only one of many channels contributing to an estimate of the overall orientation of the body and limbs. Even a $100 \%$ reduction in ankle information results in a much smaller reduction in the internal model's estimate of ankle state, and therefore does not stabilize estimator-based feedback. Normal human subjects exhibit a similar degree of robustness to altered sensory conditions (see figure 4(b)). Even though both models use the same control gains $K$, the direct feedback model lacks the robustness of human subjects or the state estimator model.

\subsection{Parameter sensitivities}

We also considered the effect of parameter variations on model behavior, by perturbing each sensory noise parameter and then recalculating the state estimation gains and SOT sway covariances (see figure 5). Each sensory parameter variation was perturbed by changing the noise-to-signal ratio. Expressed in units of decibels $(\mathrm{dB})$ of signal-to-noise ratio, $-10 \log _{10} \pi_{\text {sensor }}$, a perturbation of $+10 \mathrm{~dB}$ in signal to noise was produced by decreasing the noise-to-signal ratio by a factor of 10. The model predicts that even extreme changes in an individual sensor's precision can have very small effect on normal sway $(\kappa=1)$, because the state estimator is able to integrate useful information from the other sensors. This remains the case even with eyes closed $(\kappa=2)$, with most parameter variations resulting in sway changes of only a few per cent. The most sensitive parameter under this condition was otolith signal-to-noise ratio, where a $10 \mathrm{~dB}$ decrease produced a $21 \%$ increase in hip sway. This indicates that the model places especially greater reliance on the otoliths when visual information is lacking. Visual sway-referencing alone $(\kappa=3)$ is also predicted to have low sensitivities to parameter changes, but with one exception: the system becomes unstable when ankle proprioception signal to noise is reduced. The model compensates for poorer proprioception by placing greater weight on vision, which in this case is inaccurate. This is a recurring theme, in which the model places lower weight on poorer sensors, and greater weight on the most precise sensors, with the effects of these reweightings manifested most significantly when sensory conditions are altered.

The model exhibits a larger range of parameter sensitivities for conditions where the platform support is sway-referenced. Many parameters changes have little effect, producing sway changes of a few per cent. With normal vision $(\kappa=4)$, the only sensitivities with more than $20 \%$ change in sway were for ankle and hip proprioception, and visual translation. The ankle's sensitivity is such that a decrease in signal-to-noise ratio actually results in decreased sway, because the estimator reduces weight on the inaccurate information. This also produces the corollary effect of increased difficulty balancing on the sway-referenced platform with more precise ankle proprioception. The hip and visual translation sensitivities are of the opposite nature, where decreases in signal-to-noise result in increases in sway. When these two modalities are less effective, the model places relatively greater reliance on ankle feedback. Such 
(a) Ankle variance

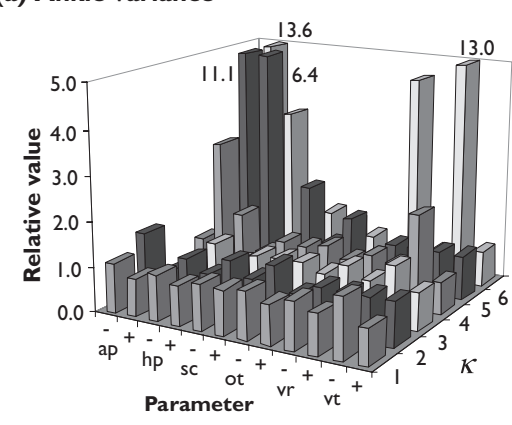

(b) Ankle-hip covariance

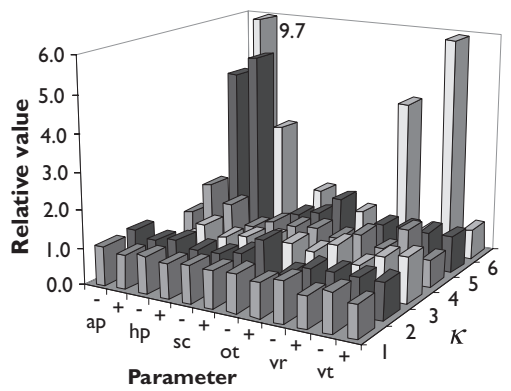

(c) Hip variance

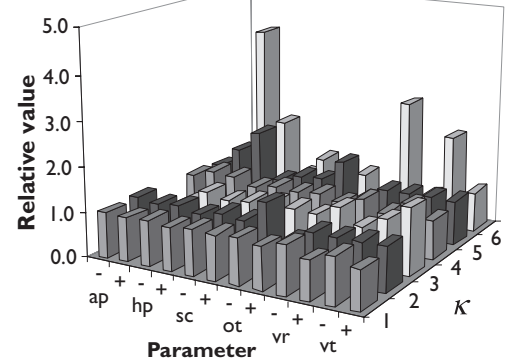

(d) State Estimator Model Covariances
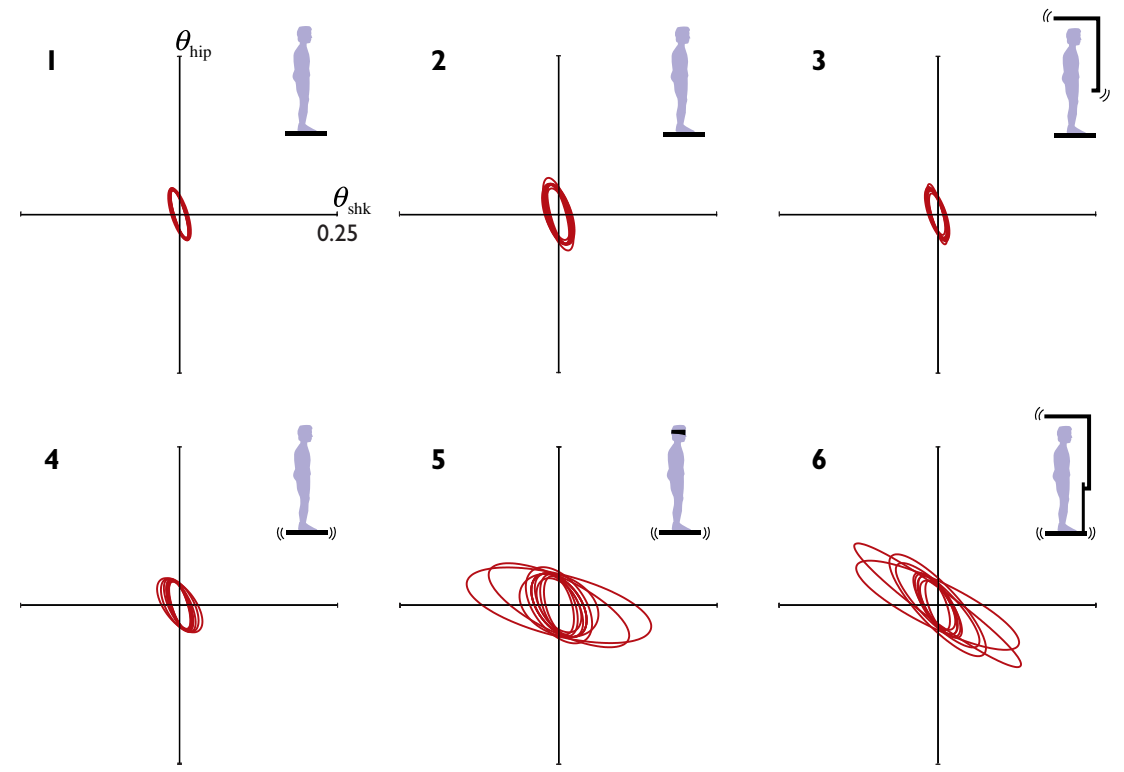

Figure 5. Parameter sensitivity study of state estimator model, showing changes in sway covariance as a function of perturbation parameter and SOT condition $(\kappa)$. Each sensor was individually perturbed with a $10 \mathrm{~dB}$ decrease (denoted by '-') or increase ('+') in signal-to-noise ratio. The state estimator model was used to optimally reweight sensors for each variation, and then predict the sway covariance under identical applied process noise. Each bar shows the resulting change in the covariance matrix: (a) ankle variance, (b) ankle-hip covariance magnitude, and (c) hip variance, relative to the nominal values of figure 4. All covariances are also shown as (d) ellipses as a function of sensory condition. The state estimator model exhibits high robustness, remaining stable for nearly all parameter values, although with greater sway under the most challenging sensory conditions. The model was highly insensitive to sensor precision in normal $(\kappa=1)$ and vision sway-referenced $(\kappa=3)$ sensory conditions. For conditions with no vision $(\kappa=2)$ or platform sway-referenced $(\kappa=5)$, there were larger insensitivities to select sensors, although sway remained small for most parameter variations. In conditions combining platform sway-referencing with eyes closed $(\kappa=5)$ or vision sway-referenced $(\kappa=6)$, most variations in sensor parameters resulted in greatly increased amounts of sway. Parameter variations caused instability in only one case, $\kappa=3$ when ankle proprioception signal-to-noise ratio is reduced (ap -); no covariance is shown for this case.

reweightings become more important when vision is removed $(\kappa=5)$, where a $10 \mathrm{~dB}$ decrease in hip proprioception causes a greater than five-fold increase in sway.

There are even greater sensitivities when visual and ankle proprioceptive sensors are both sway-referenced. Of particularly note is an instability when ankle signal to noise is decreased $(\kappa=6)$, due to the profound lack of accurate feedback. Better stability, but still high sensitivities, are also predicted for hip proprioception and visual translation and rotation, where decreases in signal-to-noise ratios cause very substantial increases in sway. Again, this is due to the estimator's compensatory strategy of placing greater weight on the remaining sensors, which do not necessarily receive accurate information.

These highest sensitivities are, however, very much in the minority. Of the 216 total parameter variations (figure 5), only $40(19 \%)$ cause more than a $20 \%$ increase or decrease in sway. For the majority of cases, there is very little sensitivity to ten-fold changes in sensor precision. This relatively small range of possible behaviors demonstrates constraints posed by the model; parameters cannot be used to attain an arbitrarily precise post hoc fit to experimental data. It also suggests why normal conditions of eyes open or eyes closed $(\kappa=$ 1 or 2) might be poor indicators of sensorimotor function 


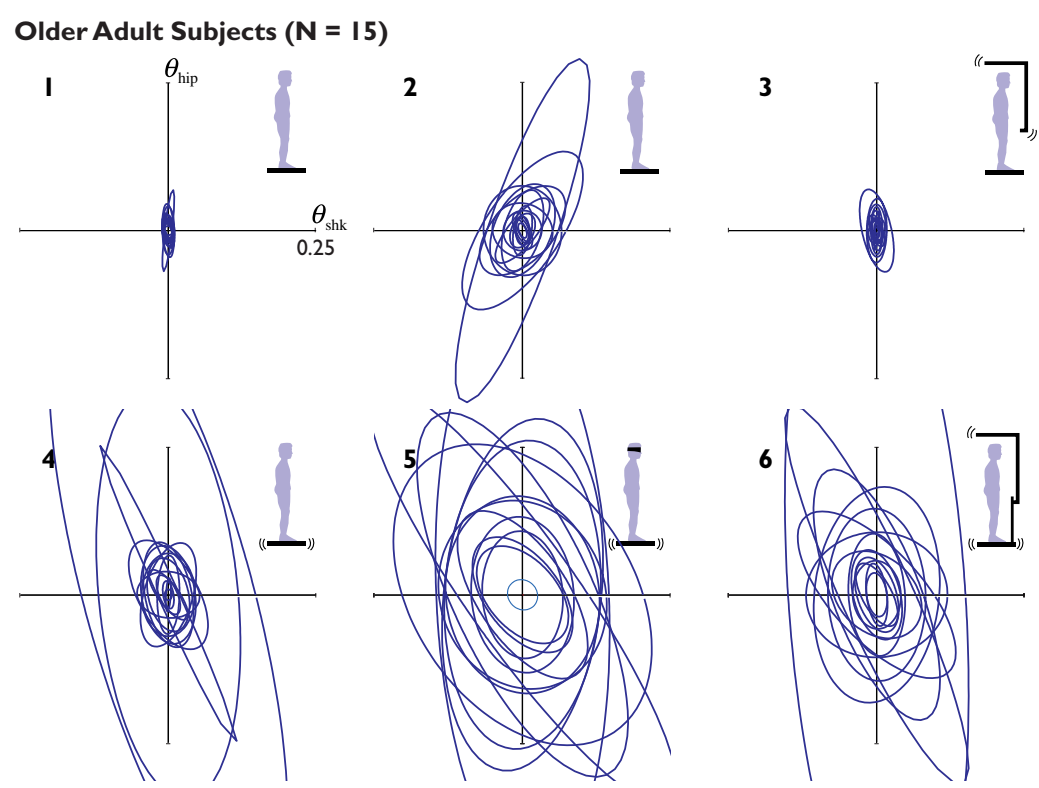

Figure 6. Experimental covariance data from healthy older adults $(N=15)$. Older adults showed very small increases in sway for the same conditions ( $\kappa=1$ and 3$)$, and intermediate amounts of variation under the other two conditions ( $\kappa=2$ and 4$)$ where only one sensory channel is missing or inaccurate. Many subjects also swayed a great deal in the last two conditions $(\kappa=5$ and 6$)$. The state estimator model also exhibited similar sensitivities in response to changes in sensor precision (signal-to-noise ratios, see figure 5(b)). Sensory and posture control degradation with age may potentially be modeled by decreases in signal-to-noise ratios in one or more sensors.

(Peterka and Black 1990), because a state estimator can successfully integrate information from the remaining sensory channels.

\subsection{Comparison with older adult subjects}

Variations in signal precision might serve as a simple model for sensory degradation during aging. Sensors degrade in many different ways with age, and by differing amounts per individual (e.g., Alexander 1994). The parameter study above only performed perturbations to a single sensor at a time, whereas the effect of aging is likely to affect multiple sensors simultaneously. However, the general sensitivity of the model, for a variety of parameter perturbations, might exhibit a similar trend of behaviors as observed in a variety of older adults. To make this comparison, we examined the parameter study covariance ellipses, along with those measured experimentally in a group of healthy older adults (aged 60-69 years, Speers et al 2002).

A visual inspection of covariances (figure 6) reveals similar trends in behavior as a function of sensory condition. The model is least sensitive to parameter changes in the conditions with normal support surface $(\kappa=1-3)$, as are the older adults. Of these three conditions, eyes closed $(\kappa=2)$ produces the most sway in both model and experiment. In the platform sway-referenced conditions ( $\kappa=4-6)$, the model exhibited larger sensitivities, especially with eyes closed or inaccurate vision. The human subjects generally swayed far more than the model, due to the model variations being restricted to single sensors. The sway observed in the model could be increased by changes of greater than $10 \mathrm{~dB}$ in individual sensors, or by simultaneous changes in multiple sensors. However, even with individual sensor variations, the model appears to exhibit broadly similar sensitivities to inaccurate or missing sensory information.

\subsection{Comparison with bilateral vestibular loss subjects}

An additional calculation was done to model response to bilateral vestibular loss, in which pairs of both canal and otolith sensors are completely dysfunctional. We modeled this dysfunction by decreasing the canal and otolith signal-to-noise ratios by $80 \mathrm{~dB}$, effectively removing any useful information from these sensors. Recalculating the state estimation gains and the resulting covariances, the model predicts remarkably small increases in sway for four SOT conditions $(\kappa=1-4)$. As long as either vision or proprioception remains accurate, the model remains stable, with the eyes closed $(\kappa=2)$ producing more sway than the others. This appears to be due to the model's greater dependence on otolith sensors with eyes closed, which was manifested (in the normal parameter sensitivity discussion above) as an increase in sway with $10 \mathrm{~dB}$ decrease in otolith signal-to-noise (see also figure 7). Extrapolating this same change to an $80 \mathrm{~dB}$ decrease, the result is still greater amounts of sway. However, the remaining two conditions $(\kappa=5,6)$ were affected to a much larger degree, with the model becoming unstable. In the absence of vestibular feedback, with inaccurate ankle proprioception and inaccurate or missing vision, there is simply insufficient data for the system to be stable.

We compared this model against sway covariances measured from three subjects with bilateral vestibular loss. These subjects were measured under the same protocol as for the older adults (Speers et al 2002). They were able 
State Estimator Model of Vestibular Loss

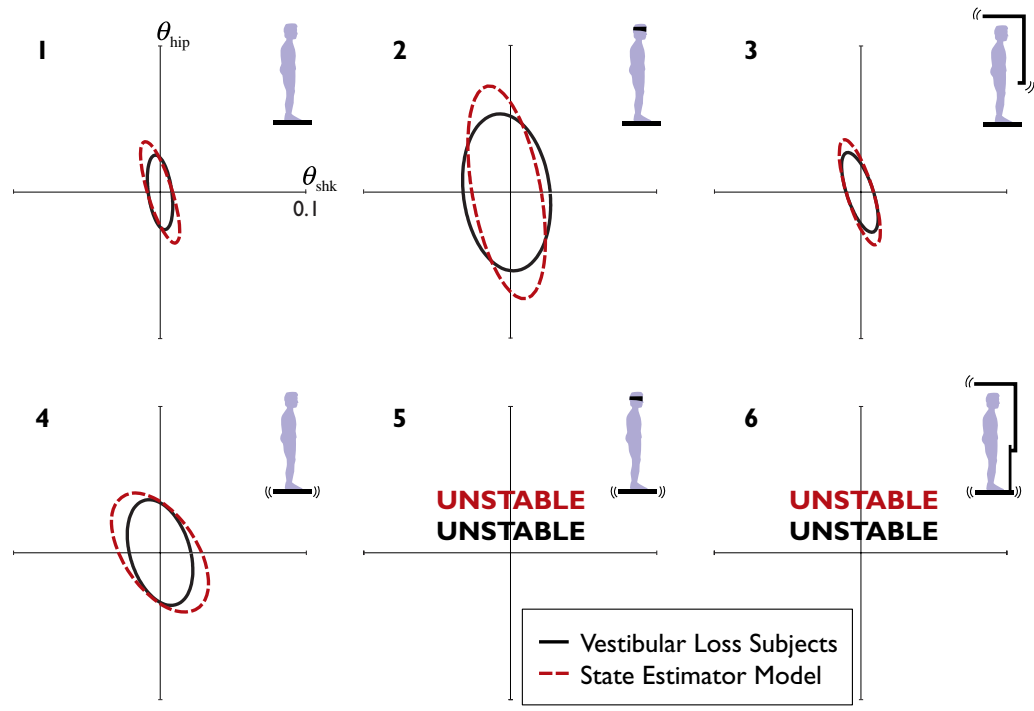

Figure 7. Comparison of sway covariances predicted by state estimator model with results from subjects with bilateral vestibular loss $(N=3)$. Vestibular loss was modeled by removal of semicircular canal and otolith feedback. Despite this change, the model was stable for the first four SOT conditions, producing substantial increases in sway only in the eyes closed $(\kappa=2)$ and platform sway-referencing $(\kappa=4)$ conditions. However, the model became unstable for the remaining conditions, where platform sway-referencing was combined with no vision $(\kappa=5)$ or inaccurate vision $(\kappa=6)$. These results compared well with larger populations of human bilateral loss subjects, for whom the same two conditions consistently produce instability (Mirka and Black 1990).

to balance well under four SOT conditions $(\kappa=1-4)$, with modest increases in sway. However, as is typical of vestibular loss patients (Mirka and Black 1990), they were unstable under the conditions with altered feedback from vision and ankle proprioception $(\kappa=5,6)$. The model agreed well with these results, both in terms of the amount of sway in the first four SOT conditions, and the instability in the last two conditions. The model displays a similar degree of robustness as vestibular loss patients to loss of one or two types of feedback, and a similar degree of instability with loss of three types of feedback.

\subsection{Summary of comparisons}

It is concluded that the model is able to reproduce much of the behavior seen experimentally in humans. It predicts high robustness to sensor degradation under normal conditions, and only slightly lower robustness when two or more sensors are rendered inaccurate. It also predicts other general changes in sway, in response to alterations in sensor information, that are consistent with experimental evidence for older adults and bilateral vestibular patients. These results are conservatively produced with only a single set of control parameters across all conditions, rather than by exploiting all of the model's degrees of freedom. It is likely that greater flexibility in parameters could improve predictions further. For example, the model responded to closure of the eyes with a larger increase in sway than exhibited by human subjects. But decreased sway could potentially be explained compensating for lack of vision by increasing the feedback control gains, thereby 'stiffening' the system. As discussed below, these behavioral predictions have certain implications regarding the organization of sensory processing in the CNS.

\section{Biological implications}

The proposed state estimator model for sensory integration has implications regarding the neural substrate for movement control, and for further experimentation of movement disorders. A neural implementation of state estimation would be expected to exhibit a convergence of multisensory information, as well as temporal filtering. Convergence is, however, not uniquely required by state estimation, because even direct feedback requires convergence of signals from many sensors. Human posture control appears to be heterogenic, with feedback from throughout the body contributing to the torque at any single joint (Barin 1989, Camana et al 1977, Speers et al 2002). This feedback property is due to the coupled dynamics of the joints. The same coupling implies that state estimation will also exhibit convergence. These two types of convergence have distinct but subtle differences. For state feedback, each joint torque will be correlated with multiple joint states, provided sensory information is accurate. The convergence in state estimation is most easily demonstrated through inaccurate sensory information, as was the case with platform swayreferencing above. These behavioral differences do not, however, lead to useful anatomical predictions. Sensory convergence is a common property of the spinal cord and higher levels of the CNS, and the state estimator model does not necessarily localize any of this convergence for the purposes of an internal model. Nor need state estimation be functionally confined to postural control alone. Feedback control of other movements such as locomotion may also require knowledge of state information, and estimation circuitry could be used for multiple purposes. 
The requirement for temporal filtering implies that the CNS may process sensory information through a set of dynamics that have similar time scales to the body and sensors. Neuronal dynamics are typically on the order of milliseconds, whereas the body has time constants on the order of seconds. A CNS implementation of state estimation must therefore assemble neurons into networks that operate on far longer time scales than a single neuron's. Such slow dynamics have long been observed in central pattern generators for locomotion, and in the velocity storage system for visual-vestibular interactions (Robinson 1981). It is therefore plausible that similar dynamics could perform state estimation. Indeed, we have proposed state estimation as an interpretation of the central pattern generator (Kuo 2002).

The internal model for state estimation also implies the need for a mechanism for adaptation. Body and sensor dynamics change throughout life, and an internal model would have to be continually updated with these changes. Such adaptation would require feedback, but in an outer loop operating at much slower time scales than posture control. Physiological evidence implicates the spinal cord and brainstem in feedback control of postural and other automatic motor tasks, and the cerebellum in learning outside of the feedback loop. The cerebellum receives a wide array of sensory data, as well as efference copy, through the mossy fiber network (Ghez 1991). It also receives error signals which appear to code differences between expected and actual input. The cerebellum does not necessarily participate in feedback control directly, but it does modify lower-level behaviors with mechanisms such as long-term depression. These could be interpreted as a substrate for adaptive state estimation (Paulin 1989). However, the present state of knowledge is insufficient to speculate beyond such an interpretation.

Aside from these neurophysiological implications, the model may also have value as a diagnostic tool. While the sensory organization test is a reliable means of diagnosing vestibular disorders, it is less sensitive for detecting systemslevel balance dysfunction in subjects suffering from other disorders, such as partial loss of proprioception or other senses. The proposed model could be used to predict the level of performance to be expected given certain degrees of sensory loss, and to predict the critical points at which such loss could result in serious instability. For example, the elderly may suffer from loss of balance due to deterioration of multiple sensors. Used in conjunction with the model, the sensory organization test could therefore potentially be used to detect deterioration at early stages and to specify appropriate rehabilitation or intervention procedures prior to loss of mobility.

\section{Conclusion}

A combined state estimator and state feedback controller reproduces and predicts the statistical behavior of quiet standing under altered sensory conditions. This model implies that the CNS forms its feedback control with an awareness of the dynamics of the system and the costs associated with certain movements. It also appears to use the equivalent of an internal model to process sensory information and form an estimate of the body state, which is then used in feedback. Such processing optimally integrates multiple sensory signals and minimizes the effects of noisy disturbances and imperfect sensors. As verified by experiment, the model predicts that loss of any single sensory modality has little effect on balance control. However, postural sway increases significantly when two of the three main modalities are disrupted. The ability to predict deterioration in performance as a function of sensory function may improve the utility of clinical balance tests and help diagnose contributors to balance dysfunction.

\section{Acknowledgments}

This work was supported in part by NIH grants DC6466 and DC02312, and the Claude Pepper Older Americans Independence Center at the University of Michigan.

\section{Appendix. Details of estimator model}

Numerical values are given for model parameters below. The body dynamics are defined in terms of matrices

$$
\begin{gathered}
G \triangleq\left[\begin{array}{l}
g_{1}^{\mathrm{T}} \\
-g_{2}^{\mathrm{T}}
\end{array}\right]=\left[\begin{array}{lr}
26.64 & -13.70 \\
-46.61 & 44.04
\end{array}\right], \\
H \triangleq\left[\begin{array}{l}
h_{1}^{\mathrm{T}} \\
\hdashline h_{2}^{\mathrm{T}}
\end{array}\right]=\left[\begin{array}{ll}
0.048 & -0.132 \\
\hdashline-0.084 & 0.3540
\end{array}\right],
\end{gathered}
$$

the rows of which are to be referred to below as $g_{1}^{\mathrm{T}}, g_{2}^{\mathrm{T}}, h_{1}^{\mathrm{T}}$ and $h_{2}^{\mathrm{T}}$. We also define a matrix $F$, which will be used to convert between joint angles $\left(\theta_{\text {shk }}\right.$ and $\left.\theta_{\text {hip }}\right)$ and segment angles $\left(\phi_{1}\right.$ and $\left.\phi_{2}\right)$ :

$$
F \triangleq\left[\begin{array}{l}
f_{1}^{\mathrm{T}} \\
-1 \\
f_{2}^{\mathrm{T}}
\end{array}\right]=\left[\begin{array}{ll}
1 & 0 \\
--1 & 1
\end{array}\right]
$$

Sensor parameters describe the dynamics of muscle spindles and vestibular organs. The muscle spindle parameters include the lead time constant $T_{\mathrm{sp}}=0.4 \mathrm{~s}$, and lag parameter $\alpha=0.15$ (Agarwal and Gottlieb 1984). Semicircular canals are parametrized by $k_{\mathrm{sc}}=0.574, \omega_{s 1}=100 \mathrm{rad} \mathrm{s}^{-1}, \omega_{s 2}=$ $0.1 \mathrm{rad} \mathrm{s}^{-1}$, and $\omega_{s 3}=0.033 \mathrm{rad} \mathrm{s}^{-1}$ (Fernandez and Goldberg 1971). Similarly, the otoliths are parametrized by $k_{\mathrm{ot}}=90$, $\omega_{o 1}=0.1$ and $\omega_{o 2}=0.2 \mathrm{rad} \mathrm{s}^{-1}$ (Young and Meiry 1968).

Several other parameters couple the body and sensor dynamics. Motion of the head is determined by ankle and hip motions, with $c_{t}^{\mathrm{T}} \triangleq\left[\begin{array}{ll}c_{t 1} & c_{t 2}\end{array}\right]=[-0.835 \mathrm{~m}-0.735 \mathrm{~m}]$ transforming angular joint accelerations into translational acceleration. The platform and body are also dynamically coupled, and the transformation from platform acceleration to joint motion described by $B_{\mathrm{p}}=\left[\begin{array}{ll}-0.13 & 0.026\end{array}\right]^{\mathrm{T}}$, derived from the body equations of motion (Park et al 2004). 
The overall system matrices are composed from the individual body and sensory matrices:

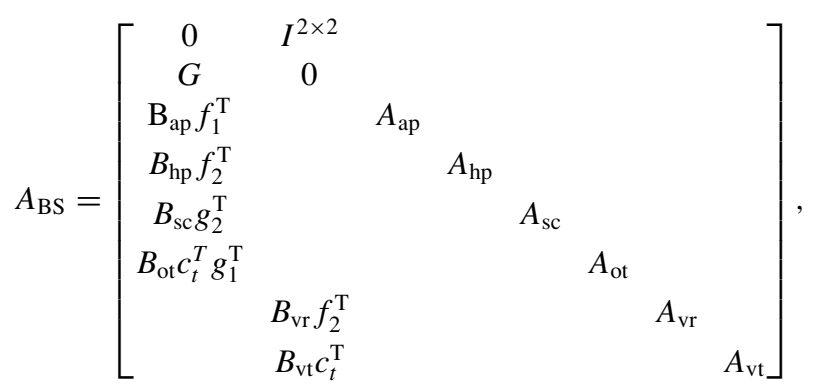

$B_{\mathrm{BS}}=\left[\begin{array}{c}0 \\ H \\ 0 \\ 0 \\ B_{\mathrm{sc}} h_{2}^{\mathrm{T}} \\ B_{\mathrm{ot}} c_{t}^{\mathrm{T}} h_{1}^{\mathrm{T}} \\ 0 \\ 0\end{array}\right]$

$C_{\mathrm{BS}}=\left[\begin{array}{cccccccc}0 & 0 & C_{\mathrm{ap}} & & & & & \\ 0 & 0 & & C_{\mathrm{hp}} & & & & \\ D_{\mathrm{sc}} g_{2}^{\mathrm{T}} & & & & C_{\mathrm{sc}} & & & \\ D_{\mathrm{ot}} c_{t}^{\mathrm{T}} g_{1}^{\mathrm{T}} & & & & & C_{\mathrm{ot}} & & \\ 0 & 0 & & & & & C_{\mathrm{vr}} & \\ 0 & 0 & & & & & & C_{\mathrm{vt}}\end{array}\right]$,

$D_{\mathrm{BS}}=\left[\begin{array}{c}0 \\ 0 \\ D_{\mathrm{sc}} h_{2}^{\mathrm{T}} \\ D_{\mathrm{ot}} c_{t}^{\mathrm{T}} h_{1}^{\mathrm{T}} \\ 0 \\ 0\end{array}\right]$

where $I^{2 \times 2}$ denotes the $2 \times 2$ identity matrix.

The control objective uses the following matrices

$Q_{\mathrm{cm}}=\left[\begin{array}{ll}0.57 & 0.17 \\ 0.17 & 0.05\end{array}\right], \quad Q_{\mathrm{up}}=\left[\begin{array}{cc}1.45 & -1.18 \\ -1.18 & 0.96\end{array}\right]$,

where only the upper-left $(2 \times 2)$ entries are shown, the rest being zero.

\section{References}

Agarwal G C and Gottlieb G L 1984 Mathematical modeling and simulation of the postural control loop: Part II. Crit. Rev. Biomed. Eng. 11 113-54

Alexander N B 1994 Postural control in older adults J. Am. Geriatr. Soc. 42 93-108

Baloh R W, Spain S, Socotch T M, Jacobson K M and Bell T 1995 Posturography and balance problems in older people J. Am. Geriatr. Soc. 43 638-44

Barin K 1989 Evaluation of a generalized model of human postural dynamics and control in the sagittal plane Biol. Cybern. 61 37-50

Borah J, Young L R and Curry R E 1988 Optimal estimator model for human spatial orientation Representation of
Three-Dimensional Space in the Vestibular, Oculomotor, and Visual Systems vol 545, ed B Cohen and V Henn (New York: The New York Academy of Sciences) pp 51-73

Bryson A E 2002 Applied Linear Optimal Control (Cambridge, UK: Cambridge University Press) p 362

Camana P C, Hemami H and Stockwell C W 1977 Determination of feedback gains for human posture control without physical intervention J. Cybern. 7 199-225

Fernandez C and Goldberg J M 1971 Physiology of peripheral neurons innervating semicircular canals of the squirrel monkey: II. Response to sinusoidal stimulation and dynamics of peripheral vestibular system J. Neurophysiol. 34 661-75

Ghez C 1991 The cerebellum Principles of Neural Science 3rd edn, ed E R Kandel, J H Schwartz and T M Jessel (New York: Elsevier) chapter 41, pp 626-46

He J, Levine W S and Loeb G E 1991 Feedback gains for correcting small perturbations to standing posture IEEE Trans. Autom. Control 36 322-32

Horak F B and MacPherson J M 1995 Postural orientation and equilibrium Handbook of Physiology (New York: Oxford University Press)

Kuo A D 1995 An optimal control model for analyzing human postural balance IEEE Trans. Biomed. Eng. 42 87-101

Kuo A D 2002 The relative roles of feedforward and feedback in the control of rhythmic movements Motor Control 6 129-45

Kuo A D, Speers R A, Peterka R J and Horak F B 1998 Effect of altered sensory conditions on multivariate descriptors of human postural sway Exp. Brain Res. 122 185-95

Kuo A D and Zajac F E 1993a A biomechanical analysis of muscle strength as a limiting factor in standing posture J. Biomech. 26 (Suppl. 1) 137-50

Kuo A D and Zajac F E 1993b Human standing posture: multi-joint movement strategies based on biomechanical constraints Prog. Brain Res. 97 349-58

Merfeld D M, Young L R, Oman C M and Shelhamer M J 1993 A multidimensional model of the effect of gravity on the spatial orientation of the monkey J. Vestib. Res. 3 141-61

Mirka A and Black F O 1990 Clinical application of dynamic posturography for evaluating sensory integration and vestibular dysfunction Neurol. Clin. 8 351-9

Nashner L M 1977 Fixed patterns of rapid postural responses among leg muscles during stance Exp. Brain Res. 30 13-24

Park S, Horak F B and Kuo A D 2004 Postural feedback responses scale with biomechanical constraints in human standing Exp. Brain Res. 154 417-27

Paulin M G 1989 A Kalman filter theory of the cerebellum Dynamic Interactions in Neural Networks: Models and Data ed M A Arbib and S-I Amari (New York: Springer)

Peterka R J and Black F O 1990 Age-related changes in human posture control: sensory organization tests J. Vestib. Res. 1 $73-85$

Robinson D A 1981 Control of eye movements Handbook of Physiology: The Nervous System vol II (Bethesda, MD: American Physiological Society) chapter 28, pp 1275-320

Speers R A, Kuo A D and Horak F B 2002 Contributions of altered sensation and feedback responses to changes in coordination of postural control due to aging Gait Posture 16 20-30

Speers R A, Shepard N T and Kuo A D 1999 EquiTest modification with shank and hip angle measurements: differences with age among normal subjects J. Vestib. Res. 9 435-44

Wiener N 1948 Cybernetics (Cambridge, MA: MIT Press)

Young L R 1981 Visual and vestibular influences in human self-motion perception The Vestibular System: Function and Morphology ed T Gualatieratti (New York: Springer) pp 393-424

Young L R and Meiry J L 1968 A revised dynamic otolith model Aerosp. Med. 39 606-8 